\title{
Cloning, Mapping, and Characterization of the Human Sorbin and SH3 Domain Containing 1 (SORBS1) Gene: A Protein Associated with c-Abl during Insulin Signaling in the Hepatoma Cell Line Hep3B
}

\author{
Wen-Hsing Lin,* Chang-Jen Huang,† Min-Wei Liu,* Hui-Min Chang,* Yann-Jang Chen,ł \\ Tong-Yuan Tai,* and Lee-Ming Chuang*,,${ }^{1}$ \\ *Department of Internal M edicine and §Graduate Institute of Clinical Medicine, College of Medicine, National Taiwan University, \\ Taipei, Taiwan; †Institute of Biological Chemistry, Academia Sinica, Taipei, Taiwan; and $\ddagger$ Center for General Education, \\ National Yang Ming University, Taipei, Taiwan
}

Received November 7, 2000; accepted March 12, 2001; published online

SH3P12/CAP/ponsin, a gene product with a sorbin homology domain and three consecutive $\mathrm{SH} 3$ domains in the carboxy-terminus, has been isolated from murine adipocytes and identified as an important adaptor during insulin signaling. Here we describe the cloning, mapping, and expression of the human homologue, termed SORBS1 (sorbin and SH3 domain containing 1). Multiple transcripts of this gene with different mR NA isoforms were observed among different tissues. Here we report 13 alternatively spliced exons, which were ascertained from the full-length cDNA cloned in adipose, liver, and skeletal muscle tissues. Among the major isoforms, the shortest, 2223-bp, open reading frame (ORF) encodes a protein with a predicted molecular weight of $81.5 \mathrm{kDa}$, while the longest, 3879-bp, ORF encodes a protein of about $142.2 \mathrm{kDa}$. This gene was mapped to human chromosome 10q23.3q24.1, which is a candidate region for insulin resistance found in Pima Indians. In human hepatoma Hep3B cells, SORBS1 was partly dissociated from the insulin receptor complex and bound to c-Abl protein upon insulin stimulation. This interaction with c-Abl was through the third SH3 domain and a possible conformational change of SORBS1 induced by insulin. Our data suggest that c-Abl oncoprotein via SORBS1 might play a role in the insulin signaling pathway. (๑) 2001 Academic Press

\section{INTRODUCTION}

SH 3P 12 bel ongs to a growing family of proteins containing a sorbin homology domain and three $\mathrm{SH} 3$ do-

\footnotetext{
${ }^{1}$ To whom correspondence and reprint requests should be addressed at the Department of Internal Medicine, National Taiwan University Hospital, 7 Chung-Shan South Road, Taipei, Taiwan. Telephone: +886-2-23123456 Ext. 5038. Fax: +886-2-23938859. E-mail: leeming@ha.mc.ntu.edu.tw.
}

mains in the $\mathrm{C}$-terminal region. This protein was first cloned by screening a mouse cDNA expression library with a SH3 binding ligand (Sparks et al., 1996) and subsequently isolated by a yeast two-hybrid system using c-Cbl as bait and a blot overlay method with the labeled 1-afadin (Ribon et al., 1998c; Mandai et al., 1999). SH $3 P 12$ was therefore denoted as CAP, a c-Cblassociated protein, or ponsin because of its interaction with both 1-afadin and vinculin in a competitive binding manner (Kurakin et al., 1998; Ribon et al., 1998c; Mandai et al., 1999). Moreover, SH3P12/CAP has been found to interact with insulin receptor, Sos, flotillin, and focal adhesion kinase (Ribon et al., 1998a,c; Baumann et al., 2000), indicating that SH 3P12/CAP might be involved in the signaling pathways to the reorganization of cytoskeleton and insulin-stimulated glucose uptake processes. I nterestingly, some insulin-sensitizing thiazolidinediones, which are specific ligand and activator for peroxisome proliferator-activated receptor $\gamma$, can increase the expression of SH3P12/CAP in adipose tissues (Ribon et al., 1998a).

Many $\mathrm{SH} 3$ domain-containing proteins such as CCrk, Nck, Abi-1, and Arg/Abl binding protein 2A (ArgBP2A) can interact with Abl and Arg via the binding of the PXXP motif and $\mathrm{SH} 3$ domain (Ren et al., 1994; Dai and Pendergast 1995; Shi et al., 1995; Wang et al., 1997; Smith et al., 1999). ArgBP2A proteins are expressed ubiquitously in human tissues and are extremely abundant in the heart. Interestingly, ArgBP2A and SH 3P12/CAP share structural homology and each protein contains a sorbin homology domain and three adjacent $\mathrm{SH} 3$ domains in the C-terminal region (Wang et al., 1997). c-Abl, a member of the Abelson nonreceptor protein tyrosine kinase family (Kruh et al., 1990; Wang, 1993), contains three conserved PXXP motifs that can serve as binding sites for SH3 domains. c-Abl play multiple roles in cellular physiology such as in cell cycle, transcription, DNA 
damage response, apoptosis, and neuronal development (Mattioni et al., 1995; Birchenall-Roberts et al., 1997; Koleske et al., 1998; Agami et al., 1999). The function of c-Abl may involve neuron development, actin cytoskel eton structure regulation, and cell-cell signaling interactions (Lewis et al., 1996; Koleske et al., 1998). Whether SH 3P 12/CAP interacts with c-Abl oncoprotein remains to be studied.

By using mRNA differential display analysis, we had also independently found that SH3P12/CAP was up regulated during adipogenesis and its expression was further enhanced by treatment with BRL 49653 in the well-differentiated 3T3-L 1 adipocytes (Lin et al., 1999). Based on these observations, it is therefore important to elucidate the gene structure and function of the human homologue, now termed SORBS1 (sorbin and SH3 domain containing 1) for better understanding of the mechanism of insulin signaling and pathogenesis of diabetes and obesity. With great advances in positional cloning for human diseases, it is also important to map the SORBS1 gene to its chromosomal location for advancing genetic information that might facilitate positional candidate gene studies for insulin resistance and associated disorders.

\section{MATERIALS AND METHODS}

EST database enquiry and primer design. By searching the human EST database (dbEST, NCBI) using the mouse SH3P12/CAP (U58883) CDNA sequence, four human EST sequences were found, i.e., Al 452468, AA873174, U 70668, and AA296029 (GenBank Accession Nos.). Ten oligonucleotide primers were synthesized for cloning of the human SORBS1 CDNA, i.e., hif (5'-GCATG AGTTC TGAAT GTGAT GGTG-3'), m345f (5'-ATGAG TTCTG AATGT GATG-3'), m1139f (5'-CTCAC TTTTT GAGCG AGGCA CAG-3'), h4f (5'GAGAT GAGAC CTGCC AGAGC C-3'), h3r (5'-TTTGG CTCTG GCAGG TCTCA TCTC-3'), m2400r (5'-TCTTA TAGAT ATAAA GG$3^{\prime}$ ), h294f (5'-AACCT TCCTT CTGAC AAGTC CC-3'), Ef (5'-AGGAC AATCC TTACA CTCCC ACC-3'), h1011r (5'-TCTTT TCGTT GGTCA GAACG GAAG-3'), and h2055r (5'-TCTTA TAGAT ACAAA GGTTT TACAT AGT-3'), as schematically shown in the Fig. 1.

Cloning and expression of SORBS1 CDNA fragments by RT-PCR. Total cellular RNA was purified from human adipose, liver, and skeletal muscle tissues using the phenol-guanidium thiocyanate extraction according to the manufacturer's protocol (TRIzol; BRL, Gaithersburg, MD). For synthesis of the first-strand CDNA, $1 \mu \mathrm{g}$ of total RNA was added to a buffer containing $5 \mathrm{mM} \mathrm{MgCl}, 50 \mathrm{mM}$ $\mathrm{KCl}, 10 \mathrm{mM}$ Tris, $\mathrm{pH}$ 8.3, $1 \mathrm{mM}$ dNTP, $0.1 \mu \mathrm{g} \mathrm{6-mer} \mathrm{degenerate}$ primers, 40 units RNasin, and 15 units SuperScript II reverse transcriptase in a total volume of $20 \mu \mathrm{l}$. The reaction mixture was incubated at $42^{\circ} \mathrm{C}$ for $1 \mathrm{~h}$ and then inactivated by heating at $70^{\circ} \mathrm{C}$ for $15 \mathrm{~min}$. The mixture was then incubated at $37^{\circ} \mathrm{C}$ for $30 \mathrm{~min}$ with 15 units of RNase $\mathrm{H}$. The CDNA mixture was then diluted to a final volume of $60 \mu \mathrm{l}$ with $\mathrm{ddH}_{2} \mathrm{O}$. For the full-length cloning, PCR was carried out in a total volume of $50 \mu \mathrm{l}$ containing $2 \mu \mathrm{l}$ of the reversetranscribed cDNA mixture, $3.5 \mathrm{mM} \mathrm{Mg}(\mathrm{OAc})_{2}, 15 \mathrm{mM} \mathrm{KOAc}, 40 \mathrm{mM}$ Tricine- $\mathrm{KOH}, \mathrm{pH}$ 8.7, $0.2 \mathrm{mM}$ dNTP, $0.2 \mu \mathrm{M}$ each $-26 \mathrm{f}$ (5'-TGCAG ACGAC TTGTC CTGCC AC-3') and 2119r (5'-AAGAT CGGTT CATGA ATGAT GCTTC-3') primers, and $1 \mu$ of $50 \times$ Advantage 2 Polymerase Mix (Clontech, Palo Alto, CA). The CDNA was amplified by denaturing at $95^{\circ} \mathrm{C}$ for $30 \mathrm{~s}$, annealed, and polymerized at $68^{\circ} \mathrm{C}$ for $4 \mathrm{~min}$ for 33 cycles. The reactions were terminated by a final extension at $68^{\circ} \mathrm{C}$ for $5 \mathrm{~min}$ and then cooled down to $4^{\circ} \mathrm{C}$. The resulting PCR fragments were then purified by agarose gel electrophoresis, subcloned into pGEMT-Easy TA vector (Promega, Madison, $\mathrm{WI}$ ), and sequenced with $\mathrm{ABI}$ Prism dye terminator cycle ready reaction kit (Perkin-Elmer, Foster City, CA).

Multitissue Northern blot analysis. The 1.5-kb EcoRI fragment from human SORBS1 CDNA (clone 2, Fig. 1) was labeled using the Rediprime II kit (Amersham Pharmacia, Piscataway, NJ ) and hybridized to a multiple-tissue Northern blot (Clontech), the membrane of which contains mRNAs derived from various tissues in an amount of $2 \mu \mathrm{g}$ each. The blot was hybridized at $65^{\circ} \mathrm{C}$ for $1 \mathrm{~h}$ in QuickHyb (Stratagene, La J olla, CA) and washed twice with $2 \times$ SSC, $0.1 \%$ SDS at room temperature for $20 \mathrm{~min}$, followed by a final wash with $0.2 \times$ SSC, $0.1 \%$ SDS at $65^{\circ} \mathrm{C}$ for $20 \mathrm{~min}$. The blot was then exposed to a Phosphor screen overnight and analyzed with a Phosphorlmager (Molecular Dynamics, Sunnyvale, CA).

BAC clone isolation and fluorescence in situ hybridization (FISH). A human BAC library (Genome Systems, St. Louis, MO) was screened according to the manufacturer's protocol by using the cDNA clone 2 (Fig. 1) as a probe. FISH experiments were performed as described previously (Pinkel et al., 1988). In brief, the slides containing metaphase chromosomes were first denatured in a solution containing $70 \%$ formamide $/ 2 \times \mathrm{SSC}$ at $72^{\circ} \mathrm{C}$ for $3 \mathrm{~min}$. The probes (from BAC clones 71P24, 144F 03, and 190A22, from Genome Systems) were labeled with biotin-dUTP by nick-translation, denatured at $76^{\circ} \mathrm{C}$ for $7 \mathrm{~min}$, and then added onto the metaphase chromosomes in $10 \mu$ l hybridization solution containing $60 \%$ formamide/ $2 \times \mathrm{SSC} / 10 \%$ dextran sulfate and $1 \mu \mathrm{l}$ human Cot-1 DNA, at $37^{\circ} \mathrm{C}$ for $18 \mathrm{~h}$. The resulting metaphase slides were interacted with FITC-conjugated avidin (Vector Laboratories, Burlingame, CA), counterstained with diamidino-2-phenylindole, and then observed under a fluorescence microscope (Zeiss Axioskop, Oberkochen, Germany).

Cell culture and cell extract preparation. Hep3B cells (HB-8064; ATCC) were grown in DMEM containing 10\% fetal bovine serum, 2 $\mathrm{mM}$ glutamine, $50 \mathrm{U} / \mathrm{ml}$ penicillin, and $50 \mathrm{U} / \mathrm{ml}$ streptomycin in a humidified incubator with $5 \% \mathrm{CO}_{2}$ at $37^{\circ} \mathrm{C}$. Cell monolayers were washed with cold PBS, after addition of $0.1 \mu \mathrm{M}$ insulin, at various time intervals as indicated. For Western blotting, the cells were lysed with a buffer containing $1 \%$ Nonidet P-40, 20 mM Tris, pH 8.0, $137 \mathrm{mM} \mathrm{NaCl}, 1 \mathrm{mM}$ phenylmethylsulfonyl fluoride, $1 \mathrm{mM} \mathrm{Na}_{3} \mathrm{VO}_{4}$, $0.15 \mathrm{U} / \mathrm{ml}$ aprotinin, and $1 \mathrm{mg} / \mathrm{ml}$ leupeptin at $4^{\circ} \mathrm{C}$. The cell extracts were then harvested by centrifugation at $12,000 \mathrm{~g}$ for $10 \mathrm{~min}$.

Expression of the glutathione S-transferase (GST) fusion proteins. Five GST fusion proteins were generated in this study. The tagged CDNA was inserted into the prokaryotic expression vector pGEX2TK (Amersham Pharmacia) and introduced into Escherichia coli BL21 (DE3) Lys for expression. Two fusion proteins, 2TK-N (nt 1-1329) and 2TK-J (containing exon J sequence), were prepared and used as antigens to generate anti-SORBSI antibodies in rabbits. The other three fusion proteins, 2TK-SH3A (nt 1330-1553), 2TK-SH3B (nt 1552-1731), and 2TK-SH3C (nt 1860-2055), containing different $\mathrm{SH} 3$ domains, were prepared for protein pull-down assay according to previous report (Chuang et al., 1993).

Antibody production, immunopreci pitation, and Western blot analyses. Rabbit polyclonal antibodies against GST-SORBS1 fusion proteins (2TK-N and 2TK-J) were prepared and partially purified through GST protein-bound glutathione Sepharose 4B (Amersham Pharmacia). The anti-phosphotyrosine, c-Abl, and anti-insulin receptor $\beta$ subunit antibodies were from Upstate Biotechnology (Lake Placid, NY) and Transduction Laboratories (San Diego, CA). Crude cellular extracts or immunoprecipitated proteins were resolved by SDS-polyacrylamide gel electrophoresis. After separation, the proteins were transferred to nitrocellul ose filter membranes and immunoblotted with specific antibodies. The proteins of interest were detected by enhanced chemiluminescence according to the manufacturer's instructions (Amersham Pharmacia). 


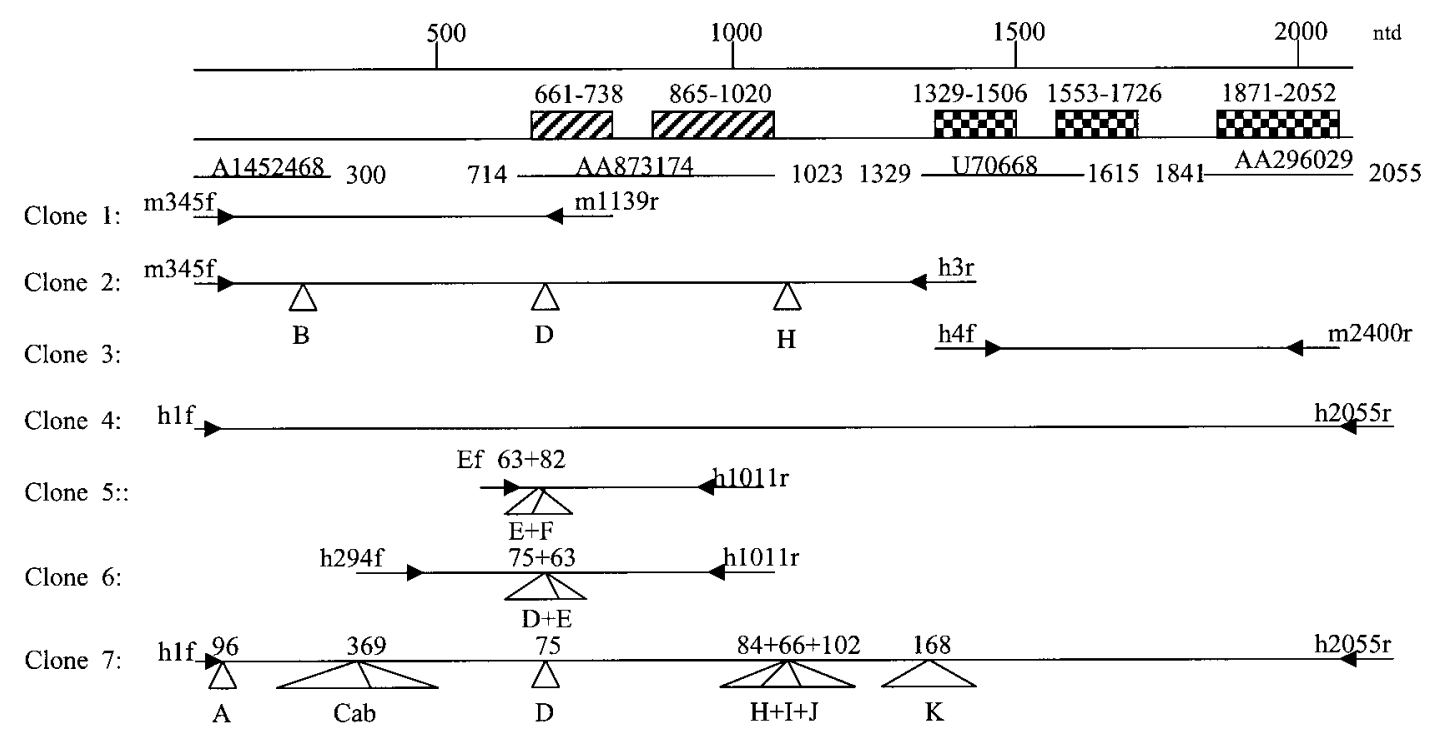

FIG. 1. Alignment of the cloned SORBS1 CDNAs. The arrowheads indicate the positions and orientations of the primers. Four homologous ESTs shown with a line and numbers on the each end indicate the positions of SORBS1 cDNA sequence (2055 bp). The striped and checkered boxes indicate the sorbin homology region and the SH3 domain, respectively. The alternative splicing exons are shown as triangles labeled from A to $\mathrm{K}$ with their lengths (basepairs) indicated above or under the triangles.

\section{RESULTS}

\section{Cloning and Sequence Analysis of SORBS1 cDNA}

By searching the human EST databank, four EST sequences (AI 452468, AA873174, AA296029, and U 70668) that were homologous to the mouse SH 3P 12/ CAP CDNA sequence (U 58883) were identified. Based on these EST sequences, 10 primers were designed to perform SORBS1 cloning from human adipose, liver, and skeletal muscle tissues. Seven cDNA clones (clones 1-7) were isolated by RT-PCR (Fig. 1). Clones 1 and 2 and clones 3 and 4 were isolated respectively from adipose and liver tissues; however, clones 5, 6, and 7 were isolated from skeletal muscle tissue. Clone 4, which contains 2055 bp had been deposited with the GenBank database under Accession No. AF 136380. Compared to mouse SH3P12/CAP, there was $87.4 \%$ homology in nucleotide sequence and an $89.3 \%$ in amino acid sequence (data not shown). Detailed analyses of the domain structure revealed a sorbin homology domain (nt 661-1020) and three consecutive SH3 domains in the C-terminus, termed SH3A, SH3B, and SH3C (nt 1329-1506, 1553-11726, and 1871-2055). To elucidate further the expression of SORBS1 in different tissues, we performed RT-PCR to isolate full-length CDNA, as shown in Fig. 2B. Of the major isoforms, we had cloned and characterized 16 (Figs. 2A and 2C). Interestingly, we found another $3^{\prime}$ donor site in exon 7 and exon 30 that resulted in exons $7 \mathrm{~b}$ and $30 \mathrm{~b}$, found in M8 and M2 clones, respectively (Fig. 2D). The sequences of clones M1, M2, and M8 had been deposited with GenBank (GenBank Accession Nos. AF 356525, AF 356526, and AF 356527). With sequence comparison by homology search, the nucleotide sequences of the isolated exons were novel and without significant similarities to those deposited with GenBank, except that exon $\mathrm{H}$ was homologous to Arg/Abl binding protein 2A and exon $\mathrm{K}$ was homologous to $\mathrm{F}$ as-ligand binding protein 2. Interestingly, exon F (82 bp) found in clone 5 (Fig. 1) was homologous to an EST sequence (BF 959891) that was from a human breast tumor.

\section{Genomic Organization of the SORBS1 Gene}

To characterize the human SORBS1 genomic structure, we had used the sequences AF 136380, AF 356525, AF 356526, AF 356527, and clone 5 to search the NCBI human genomic sequence databank. Based on the search and alignment, the coding region of this gene was encoded by 34 exons (separated by 33 introns) spanning a region of more than $120 \mathrm{~kb}$ (Fig. 2A). As shown in Fig. $2 A$, intron size was derived from the human genomic sequences (NCBI). But, four intron sizes, shown as the gaps in the Fig. $2 \mathrm{~A}$, could not be obtained from the search.

\section{Tissue Distribution of SORBS1 Transcripts}

To determine the tissue-specific distribution, Northern blot analysis was performed on a human multipletissue blot. To avoid nonspecific hybridization due to the conserved SH3 domains, we use CDNA clone 2 (Fig. 1 ) as probe. The result showed that human SORBS1 was ubiquitously expressed in all the human tissues, including brain, heart, skeletal muscle, colon, thymus, spleen, kidney, liver, small intestine, placenta, lung, and peripheral blood leukocytes. However, SORBS1 was more abundantly expressed in the heart and skeletal muscles than in the other tissues (Fig. 3). More- 
A

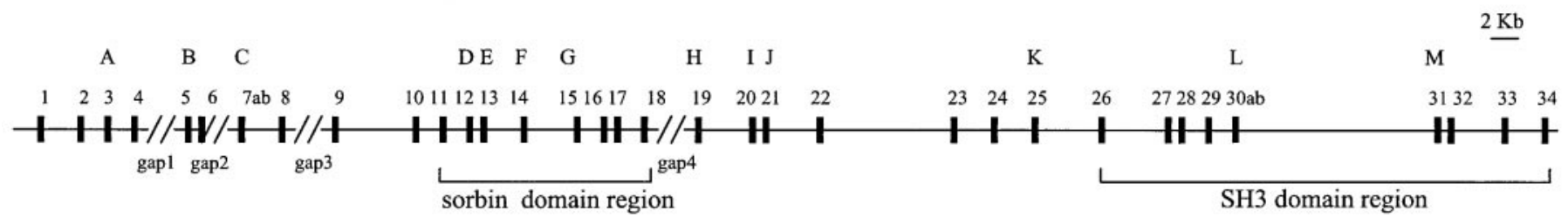

B

(kb) $\quad$ A $\quad$ L $\quad M$

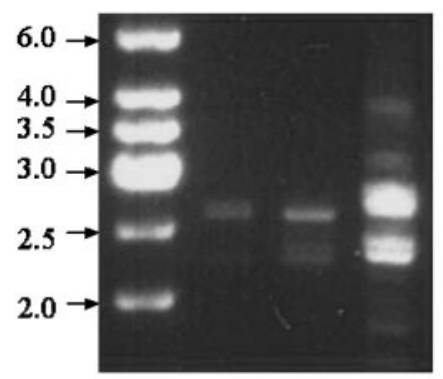

C

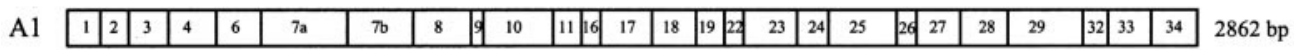

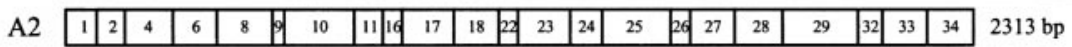

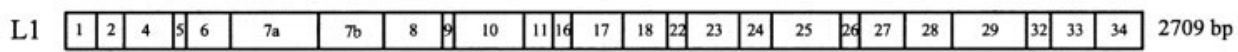

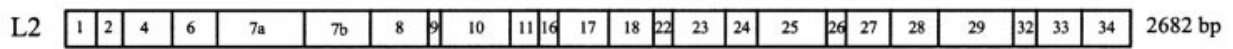

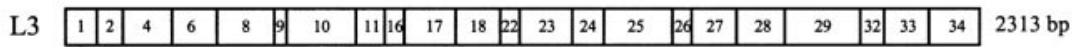

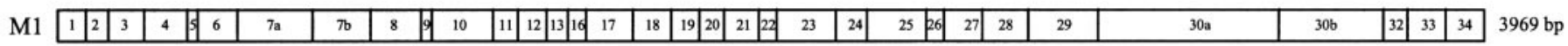

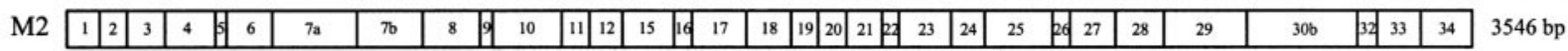

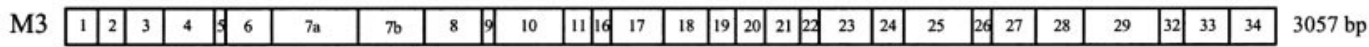

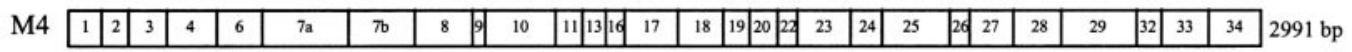

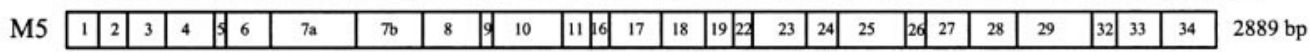

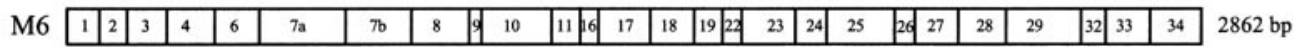

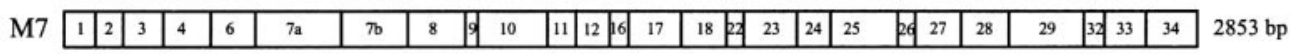

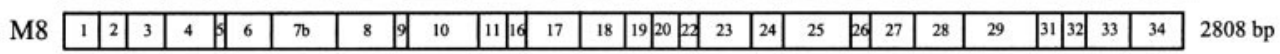

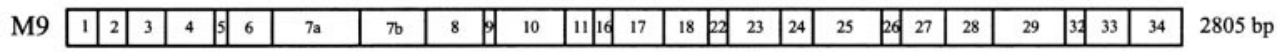

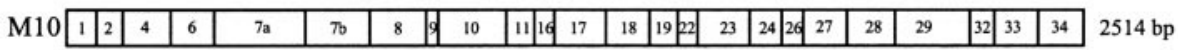

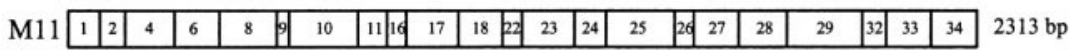

FIG. 2. Genomic organization and mRNA expression of the SORBSI gene. (A) Genomic structure of SORBS1. Exons are depicted as black boxes. The splicing exons named $A$ to $M$ are depicted over their respective boxes. The sorbin domain is encoded by exons $11,16,17$, and 18 and the SH3 domain by exons 26, 27, 28, 29, 32, 33, and 34. The sizes of introns 4, 6, 8, and 18 are not ascertained. (B) The major RT-PCR fragments generated from the CDNA of human adipose (lane A), liver (lane $\mathbf{L}$ ), and skeletal muscle (lane $\mathbf{M}$ ) tissues. The molecular size markers are indicated on the left. (C) The exon organization of the 16 full-length clones isolated from adipose (A1 and A2), liver (L 1-L3), and skeletal muscle (M 1-M 11) tissues. (D) The sequence of the genomic DNA fragments that contain exons 7ab and 30ab. The lowercase letters indicate intron sequence and uppercase letters denote exons 7ab and 30ab, where exons 7b and 30b are shown in boldface.

over, in heart, skeletal muscle, brain, and liver, multiple transcripts containing various alternatively spliced exons were detected, consistent with a previous report showing that SORBS1 had several splicing variants (Mandai et al., 1999).

\section{Chromosomal Localization of SORBS1}

For chromosomal mapping of human SORBS1, a human BAC library was screened by using the SORBS1 cDNA. Three clones, 71P24, 144F 03, and 190A22, were 
D

Exon $7 \mathrm{ab}$ ( $369 \mathrm{bp}$ )

aacgacccce ttatttccac agGATGCCAC TTCCTCCAGT GCAGCCCAGC CGGAGGTAAT AGTTGTCCCT CTCTACCTGG TTAATACTGA CAGAGGGCAA GAAGGCACTG CCAGACCTCC AACACCTCTG GGGCCTCTTG GCTGCGTCCC CACAATCCCA GCGACTGCCT CTGCCGCCTC ACCTCTGACC TTCCCGACTC TAGATGATTT CATTCCCCCT CATCTGCAGA GGTGGCCCCA CCACAGCCAG CCAGCCCGCG CCTCTGGCTC CTTTGCCCCC ATTAGCCAGA CGCCACCATC CTTCTCACCA CCACCTCCGC TGGTCCCTCC TGCCCCGGAG GACCTCCGCA GAGTCTCGGA GCCTGACCTC ACGGGAGCTG TTTCGAGTAC Cgtaagcttg ctggactcce ctcctcacgg

Exon 30ab ( $744 \mathrm{bp})$

tctttgtte tcctctctt tcttctttt aagTTTTCCA GTCACAGCAA GCTCATCACG CCAGCCCCCT CATCTCTGCC CCACTCCCGC CGAGCCCTGT CCCCCGAGAT GCACGCTGTC ACCTCTGAGT GGATCTCACT GACTGTGGGG GTCCCAGGCA GGCGTTCTCT GGCCCTGACC CCACCCTTGC CTCCTCTGCC AGAGGCTTCT ATCTATAACA CTGACCACCT CGCCTTGTCA CCAAGGGCCA GTCCCTCCCT GTCTCTCAGC CTCCCCCATT TGAGTTGGTC AGATCGTCCC ACCCCACGAT CAGTAGCTTC TCCACTGGCC CTACCTTCCC CACACAAAAC CTACTCCCTA GCACCTACTT CCCAGGCCTC CCTTCACATG AATGGAGACG GTGGTGTCCA CACGCCATCT TCAGGCATCC ACCAAGATAG CTTCTTGCAG CTGCCGCTGG GGAGCTCTGA TAGTGTCATC TCCCAGCTTA GTGATGCCTT TAGCAGCCAG AGCAAGAGGC AGCCATGGCG CGAAGAGAGT GGACAATATG agAgGAaAgC AGAGAGGgGg GCAGGCGAAA GAGGCCCTGG TGGACCCAAG ATCTCTAAGA AGAGCTGCTT GAAGCCTTCA GACGTGGTCA GGTGCCTGAG TACTGAACAG AGACTCTCAg ATCTCAACAC CCCTGAGGAG AGCCGGCCCG GCAAGCCCCT GGGTAGCGCT TTTCCAGGAA GTGAGGCTGA GCAGACAGAG CGgCATAGAg GTGGCGAGCA GGCGGGGAGG AAAGCTGCTC GGAGAGGTGG GAGCCAGgta ggactgcagC atgecgegtg tgcacttggC FIG. 2-Continued

isolated and confirmed by sequencing with $\mathrm{h} 4 \mathrm{f}$ primer and Southern blotting (data not shown). These BAC clones were used as probes for doing the FISH experiment; 100 mitotic lymphocyte spreads all showed specific signals on one pair of chromosomes (Fig. 4). The SORBS1 gene was clearly mapped to human chromosome 10q23.3-q24.1 (Fig. 4). Recently, the mouse SH3P12/CAP (SH3d5) had been mapped to mouse chromosome 19 (35.50-37.50 cM), the syntenic region of human chromosome 10q23.1-q24.1 (Lee et al., 1999).

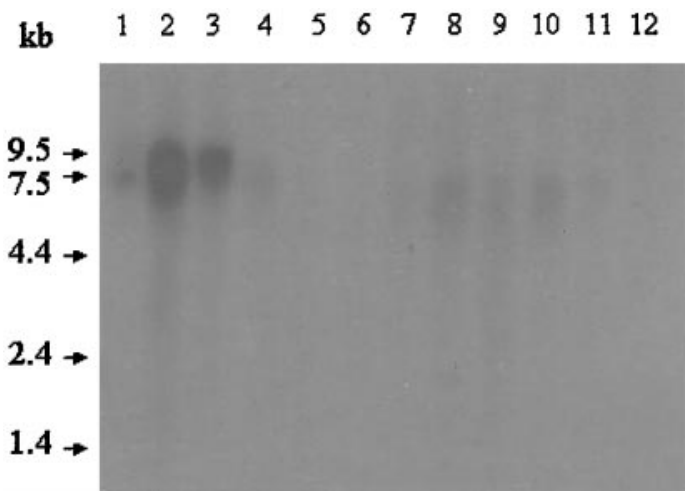

FIG. 3. Tissue distribution of the SORBS1 mRNA. The human mRNA blot was probed with radioactive SORBS1 CDNA (clone 2). Lane $\mathbf{1}$ indicates brain; 2, heart; $\mathbf{3}$, skeletal muscle; 4, colon; 5, thymus; $\mathbf{6}$, spleen; $\mathbf{7}$, kidney; $\mathbf{8}$, liver; $\mathbf{9}$, small intestine; 10, placenta; 11, lung; and 12, peripheral blood leukocytes. Size markers are indicated on the left.
Interactions of SORBS1 with I nsulin Receptor and c-Abl

Sequence comparisons of SORBS1, especially the isoforms containing exon $\mathrm{H}$, revealed a high homology with ArgBP2A, a protein that interacts with c-Abl oncoprotein (Fig. 5A). The c-Abl protein has three PXXP motifs that actually serve as an SH3 domain binding site to interact with some $\mathrm{SH} 3$ domain-containing protein; one of the PXXP motifs (amino acids 628-641) is a sequence that is highly conserved with the PXXP motif sequence of $\mathrm{C}-\mathrm{Cbl}$ and Sosl (Fig. 5B). It has been known that the PXXP motifs of $\mathrm{c}-\mathrm{Cbl}$ and Sos1 provide the binding site for the third SH3 domain of SH3P12/ CAP protein (Kurakin et al., 1998). To study further the candidate protein that will interact with SORBS1 in insulin signaling, we then performed immunoprecipitation to examine the interaction between SORBS1 with c-Abl and insulin receptor in a human hepatoma cell line, Hep3B. Interestingly, SORBS1 was coprecipitated with the insulin receptor $\beta$ subunit but not c-Abl in the basal state. After insulin stimulation, SORBS1 tended to dissociate from the receptor complexes (Fig. 6), as similarly reported in the 3T3-L 1 adipocytes ( $\mathrm{Ri}$ bon et al., 1998c). As immunoprecipitated with antiSORBS1 antibody after insulin stimulation, the amount of the binding of c-Abl oncoproteins to the SORBS1 proteins was remarkably increased (Fig. 7A). To map further the region responsible for the interac- 
A

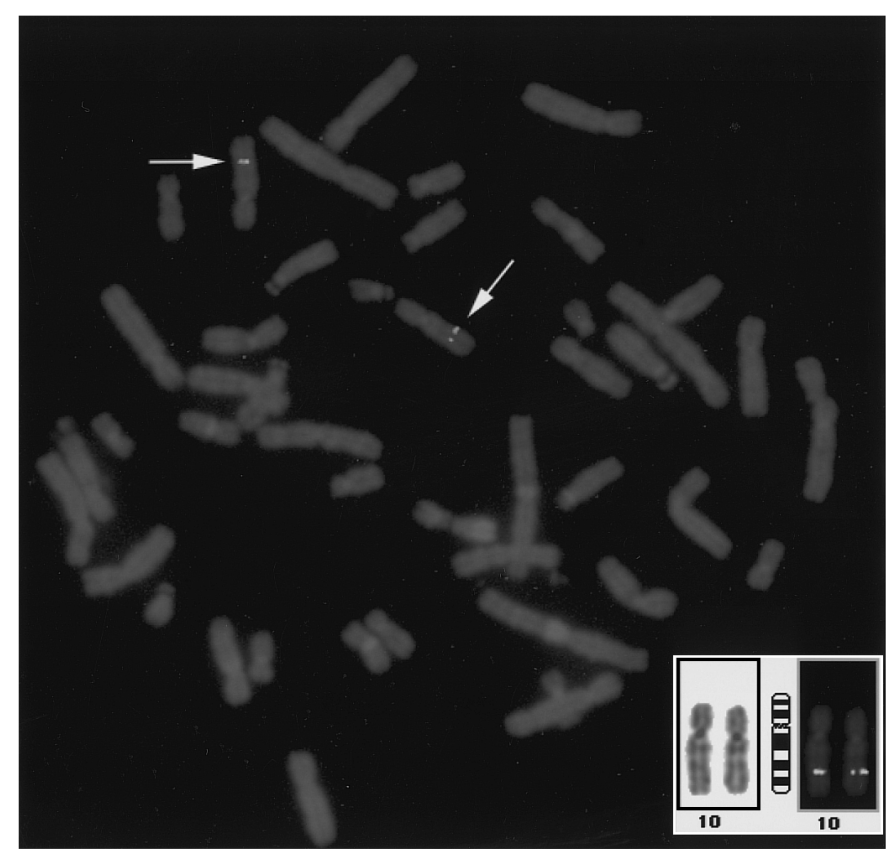

B

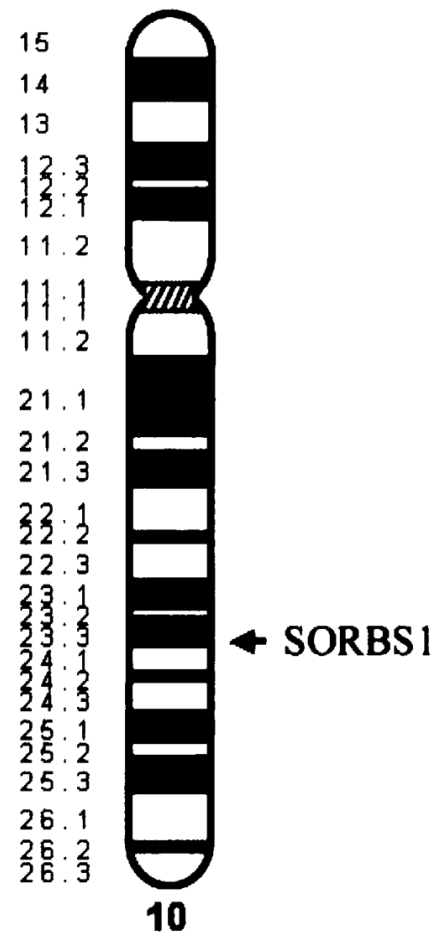

FIG. 4. Fluorescence in situ hybridization of SORBS1. (A) A BAC clone containing human SORBS1 gene was hybridized to normal human metaphase chromosomes as described under Materials and Methods. Arrows point to the site of hybridization of the SORBS1 probe; both sister chromatids show hybridization at that site. (B) I diogram of human chromosome 10 shows the map of localization of the human SORBS1 gene at 10q23.3- q24.1 (arrow).

tion with c-Abl oncoprotein, we expressed the GSTfusion proteins containing the three $\mathrm{SH} 3$ domains of the SORBSI gene separately. We found that the third $\mathrm{SH} 3$ domain, namely SH3C, was responsible for the binding of c-Abl (Fig. 7B). It is interesting to note that the isolated GST-SH3C protein was capable of binding the c-Abl proteins present both in the control and in the insulin-stimulated cell lysates, implying that the binding between SORBS1 and C-Abl inside the intact cells requires a modification of SORBS1 protein upon insulin stimulation.

\section{DISCUSSION}

We have identified the human SORBS1 cDNA from adipose, liver, and skeletal muscle tissues by RT-PCR. SORBS1 is a member of the novel SH3P12/ArgBP2A/ vinexin adaptor family. In this study, we isolated 13 alternatively spliced exons and demonstrated that the isoforms with these exons in the three insulin-sensitive tissues are different. The observation of multiple splicing isoforms of SORBS1 is similar to the discovery of more than 12 variants found in mouse tissues (Mandai et al., 1999). The expression of SORBS1 is ubiquitous in all tissues, but it is preferentially expressed in the heart and skeletal muscle. The high expression of SORBS1 in heart is similar to that of ArgBP2A, an Abl/Arg tyrosine kinase binding protein (Wang et al., 1997). Therefore, we speculate that SORBS1 might exert a special function related to ArgBP2A in heart and skeletal muscle tissues.

SORBS1 protein is structurally related to ArgBP2A and vinexin, a vinculin binding protein. This family of proteins can interact with vinculin, 1-afadin, and other associated proteins. The specific SORBS1 isoforms, i.e., those containing spliced exon $\mathrm{H}$, have a high homology to ArgBP2A. Based on the sequence similarity between SORBS1 and ArgBP2A, and the consensus motifs (three PXXP motifs) identified in C-Abl for SH3 domain binding, we investigated the interaction between SORBS1 with insulin receptor and c-Abl in Hep3B cells. We found that SORBS1 associated with insulin receptor $\beta$ subunit but not c-Abl in the unstimulated state. However, following insulin stimulation, SORBS1 was dissociated from insulin receptor while the binding to c-Abl was increased. Interestingly, the binding between SORBSI and c-Abl was through the third SH3 domain (SH3C) in the C-terminus, as demonstrated in the GST-SH3C fusion protein pull-down assays with the cell lysates either from basal or after insulin stimulation. Taken together, our data suggest that some structural modification occurs in SORBS1 rendering it capable of binding c-Abl in intact cells when treated with insulin.

Different cellular functions might occur due to the different subcellular localizations of these interacting proteins. Previously, it is known that CAP/SH3P 12 protein can associate with insulin receptor, flotillin, c-Cbl oncoprotein, Sos adaptor, and focal adhesion kinase (FAK), suggesting that SORBS1 might be involved in the insulin signaling pathway and cytoskeleton organization. It has been demonstrated that nuclear localization of c-Abl plays a role in controlling 


\section{A}

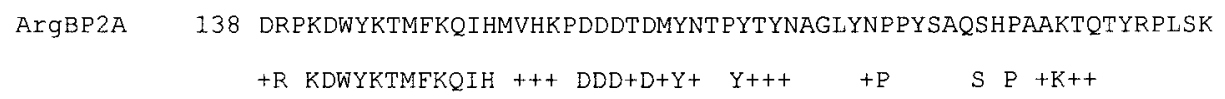

SORBS1 225 ERSKDWYKTMFKQIHKLNR-DDDSDLYSPRYSESEDTKSP----LSVPRSKSEM---.--

198 SHSDNSPNAFKDASS PVPPPHVPPPVPPLRPRDRSSTEKHDWDP PDRKVDTRKFRSEPRS

$\mathrm{S}+\mathrm{D}+\mathrm{A}+\mathrm{P}+\mathrm{P}+\mathrm{SS}+\mathrm{E}++\mathrm{DW}+\mathrm{PPD}+\mathrm{KVDTRK}+\mathrm{R}+\mathrm{EP}+\mathrm{S}$

274 SYIDGEKVVKRSATLPLPARSS-------SLKSSSERNDWEPPDKKVDTRKYRAEPKS

258 I FEYEPGKSS I LQHERPTDRINPDD IDLENEPWYKEFSELEFGRP

$\mathrm{I}+\mathrm{EY}+\mathrm{PGKSS}+\mathrm{L}+\mathrm{E}++\mathrm{I}+\mathrm{P}++\mathrm{IDL}+\mathrm{NEPWYKFFSELEFG}+\mathrm{P}$

325 IYEYQPGKSSVLTKEKMSRDISPEEIDLKNEPWYKFFSELEFGKP

B

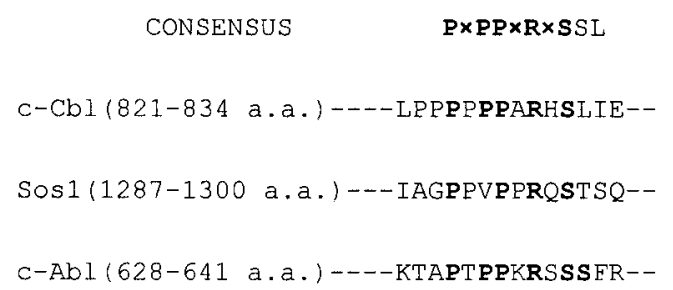

FIG. 5. (A) Comparison of primary protein structure of ArgBP2A with SORBS1 containing exon $\mathrm{H}$ sequences. The amino acid sequence of exon $\mathrm{H}$ is indicated in bold. (B) Alignment of the primary protein structure of c-Cbl, Sos, and c-Abl with the consensus sequence.

cell cycle, stress response, DNA repair, transcriptional activity, and apoptosis. However, the function of cytoplasmic c-Abl is far less understood. It has been shown that cytoplasmic c-Abl is associated with the actin cytoskeleton through its C-terminal actin-binding domain following growth factor and integrin signaling (Lewis et al., 1996; Plattner et al., 1999; Lanier and Gertler, 2000). SH3P12/CAP protein interacts with FAK through its middle SH3B domain (Ribon et al.,

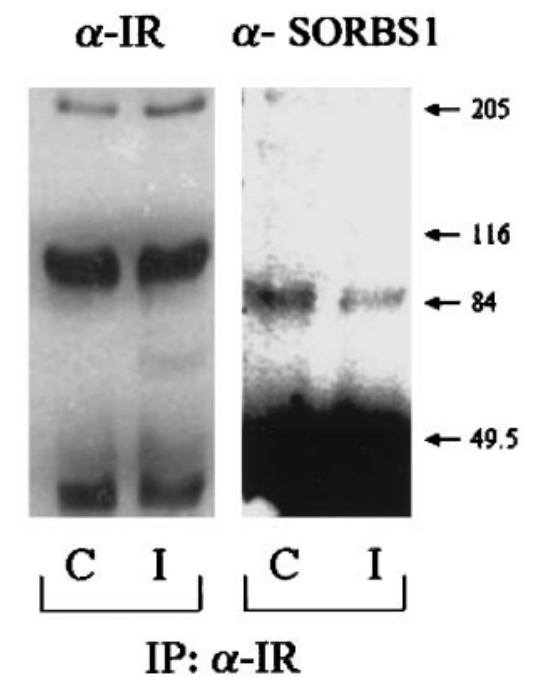

FIG. 6. Interaction of insulin receptor and SORBS1. Total celluIar extracts from the Hep3B cells, control (C) and insulin stimulated for 5 min (I), were first immunoprecipitated with anti-IR antibody and then blotted with anti-IR (left) and anti-SORBS1 (right). Arrows (right) indicate the molecular weight markers in kDa. 1998a). Taken together, our data suggest a possibility that the interaction of FAK and actin is via the interaction of SORBS1 and C-Abl.

In the FISH experiments, we found that the SORBSI gene was a single-copy gene and mapped to the human chromosome 10q23.3- q24.1 region. Recently, an interesting chromosomal region linked to insulin action was also mapped to the 10q23-q24 region in the Pima Indian population (Pratley et al., 1998). However, a search for mutation of a candidate gene, the protein targeting to glycogen/PPP1R5, in this region had been pursued in vain. No contribution of this gene to insulin sensitivity was concluded (Permana et al., 1999). Since SH3P 12/CAP was associated with insulin receptor in several insulin-sensitive tissues (Ribon et al., 1998c, and present study), and up regulated in 3T3-L 1 adipocytes/adipose tissues after treatment with insulin sensitizer (Ribon et al., 1998b; Lin et al., 1999), collectively, these data suggest that SORBS1 represents a positional and functional candidate gene for insulin sensitivity.

In conclusion, the SORBSI gene is expressed in all of tissues but with a tissue-specific pattern. We have cloned and identified different isoforms that were due to the alternative splicing mechanism. Interaction between SORBS1 protein with c-Abl is insulin-dependent and via its C-terminal SH3 domain, implying a role for SORBS1/c-Abl interaction in the insulin signaling pathway. SORBS1 is mapped to the chromosome 10q23.3-q24.1 region, a candidate region for insulin sensitivity found in Pima Indians. Thus, SORBS1 


\section{Immunoblots}

\section{$\alpha$-SORBS1 $\alpha$-c-Abl}

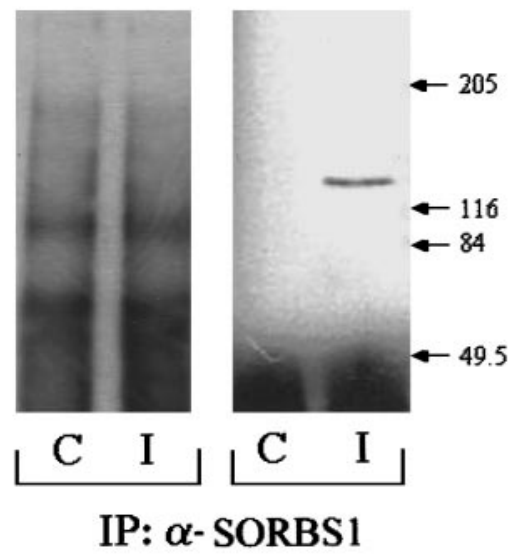

B

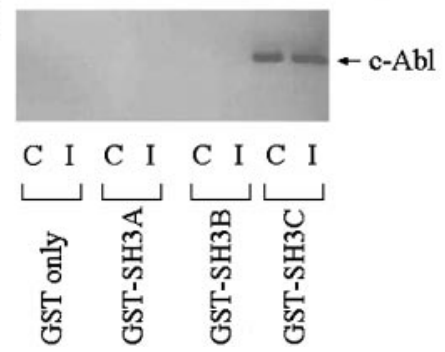

FIG. 7. Interaction of SORBSI and c-Abl. (A) Immunoprecipitation. Total cellular extracts from the Hep3B cells, control (C) and insulin stimulated for $5 \mathrm{~min}$ (I), were immunoprecipitated with antiSORBS1 antibody and immunoblotted with anti-SORBS1 (left) and anti-c-Abl (right). Arrows (right) indicate the molecular weight markers in kDa. (B) GST-fusion protein pull-down. Total cellular extracts from the Hep3B cells, control (C) and insulin stimulated for 5 min (I), were incubated with GST alone and GST fusion proteins of the three SH3 domains (SH3A, SH3B, and SH3C) of the SORBS1. The bound proteins were then transferred and blotted with anti-cAbl antibody (indicated by arrow on the right).

serves as a positional and functional candidate for insulin sensitivity and the associated phenotype such as obesity and/or diabetes mellitus.

\section{ACKNO WLEDGMENTS}

The authors thank Ms. Ya-Hui Huang for her technical assistance. This study was supported by grants from the National Science Council of the R.O.C. (NSC 87-2314-B-002-069-M21 and 88-2314-B-002077-M21), Department of Education (89-B-FA01-1-4), and National Taiwan University Hospital (NTUH-S861056-A12).

\section{REFERENCES}

Agami, R., Blandino, G., Oren, M., and Shaul, Y. (1999). Interaction of c-Abl and p73alpha and their collaboration to induce apoptosis. Nature 399: 809-813.

Baumann, C. A., Ribon, V., Kanzaki, M., Thurmond, D. C., Mora, S., Shigematsu, S., Bickel, P. E., Pessin, J. E., and Saltiel, A. R. (2000). CAP defines a second signalling pathway required for insulin-stimulated glucose transport. Nature 407: 202-207.
Birchenall-Roberts, M. C., Yoo, Y. D., Bertolette, D. C., 3rd, Lee, K. H., Turley, J. M., Bang, O. S., Ruscetti, F. W., and Kim, S. J . (1997). The p120-v-Abl protein interacts with E2F-1 and regulates E2F-1 transcriptional activity. J . Biol. Chem. 272: 8905- 8911.

Chuang, L. M., Myers, M. G., J r., Backer, J. M., Shoelson, S. E., White, M. F., Birnbaum, M. J ., and Kahn, C. R. (1993). Insulinstimulated oocyte maturation requires insul in receptor substrate 1 and interaction with the $\mathrm{SH} 2$ domains of phosphatidylinositol 3-kinase. Mol. Cell. Biol. 13: 6653-6660.

Dai, Z., and Pendergast, A. M. (1995). Abi-2, a novel SH3-containing protein interacts with the c-Abl tyrosine kinase and modulates c-Abl transforming activity. Genes Dev. 9: 2569-2582.

Koleske, A. J ., Gifford, A. M., Scott, M. L., Nee, M., Bronson, R. T., Miczek, K. A., and Baltimore, D. (1998). Essential roles for the Abl and Arg tyrosine kinases in neurulation. Neuron 21: 1259-1272.

Kruh, G. D., Perego, R., Miki, T., and Aaronson, S. A. (1990). The complete coding sequence of arg defines the Abelson subfamily of cytoplasmic tyrosine kinases. Proc. Natl. Acad. Sci. USA 87: 58025806.

Kurakin, A., Hoffman, N. G., and Kay, B. K. (1998). Molecular recognition properties of the C-terminal $\mathrm{SH} 3$ domain of the $\mathrm{Cbl}$ associated protein, CAP. J . Pept. Res. 52: 331-337.

Lanier, L. M., and Gertler, F. B. (2000). From Abl to actin: Abl tyrosine kinase and associated proteins in growth cone motility. Curr. Opin. Neurobiol. 10: 80-87.

Lee, C. G., Morse, H. C., 3rd, Kay, B. K., and Kozak, C. A. (1999). Genetic mapping of eight $\mathrm{SH} 3$ domain genes on seven mouse chromosomes. Mamm. Genome 10: 402- 404.

Lewis, J. M., Baskaran, R., Taagepera, S., Schwartz, M. A., and Wang, J. Y. (1996). Integrin regulation of c-Abl tyrosine kinase activity and cytoplasmic-nuclear transport. Proc. Natl. Acad. Sci. USA 93: 15174-15179.

Lin, W. S., Chang, H. M., Tai, T. Y., and Chuang, L. M. (1999). Effect of thiazolidinedione on gene expression in NIH 3T3-L 1 adipocytes. Diabetes 48 (Suppl. 1): A217.

Mandai, K., Nakanishi, H., Satoh, A., Takahashi, K., Satoh, K., Nishioka, H., Mizoguchi, A., and Takai, Y. (1999). Ponsin/CAP: An L-afadin- and vinculin-binding protein localized at cell-cell and cell-matrix adherens junctions. J . Cell Biol. 144: 1001-1017.

Mattioni, T., J ackson, P. K., Bchini-Hooft van Huijsduijnen, O., and Picard, D. (1995). Cell cycle arrest by tyrosine kinase Abl involves altered early mitogenic response. Oncogene 10: 1325-1333.

Permana, P. A., Luczy-Bachman, G., and Bogardus, C. (1999). Protein targeting to glycogen/PPP1R5: Screening of coding and flanking genomic regions for polymorphisms and association analysis with insulin action in Pima Indians. Biochem. Biophys. Res. Commun. 258: 184-186.

Pinkel, D., Landegent, J ., Collins, C., Fuscoe, J ., Segraves, R., Lucas, J ., and Gray, J. (1988). Fluorescence in situ hybridization with human chromosome-specific libraries: Detection of trisomy 21 and translocations of chromosome 4. Proc. Natl. Acad. Sci. USA 85: 9138-9142.

Plattner, R., Kadlec, L., DeMali, K. A., Kazlauskas, A., and Pendergast, A. M. (1999). c-Abl is activated by growth factors and Src family kinases and has a role in the cellular response to PDGF. Genes Dev. 13: 2400-2411.

Pratley, R. E., Thompson, D. B., Prochazka, M., Baier, L., Mott, D., Ravussin, E., Sakul, H., Ehm, M. G., Burns, D. K., Foroud, T., Garvey, W. T., Hanson, R. L., Knowler, W. C., Bennett, P. H., and Bogardus, C. (1998). An autosomal genomic scan for loci linked to prediabetic phenotypes in Pima Indians. J. Clin. Invest. 101: 1757-1764.

Ren, R., Ye, Z. S., and Baltimore, D. (1994). Abl protein-tyrosine kinase selects the Crk adapter as a substrate using SH3-binding sites. Genes Dev. 8: 783-795.

Ribon, V., Herrera, R., Kay, B. K., and Saltiel, A. R. (1998a). A role for CAP, a novel, multifunctional Src homology 3 domain-contain- 
ing protein in formation of actin stress fibers and focal adhesions. J . Biol. Chem. 273: 4073-4080.

Ribon, V., J ohnson, J . H., Camp, H. S., and Saltiel, A. R. (1998b). Thiazolidinediones and insulin resistance: Peroxisome proliferator activated receptor gamma activation stimulates expression of the CAP gene. Proc. Natl. Acad. Sci. USA 95: 14751-14756.

Ribon, V., Printen, J. A., Hoffman, N. G., Kay, B. K., and Saltiel, A. R. (1998c). A novel, multifunctional c-Cbl binding protein in insulin receptor signaling in 3T3-L 1 adipocytes. Mol. Cell. Biol . 18: 872- 879.

Shi, Y., Alin, K., and Goff, S P. (1995). Abl-interactor-1, a novel SH3 protein binding to the carboxy-terminal portion of the Abl protein, suppresses v-abl transforming activity. Genes Dev. 9: 2583-2597.
Smith, J . M., Katz, S., and Mayer, B. J . (1999). Activation of the Abl tyrosine kinase in vivo by Src homology 3 domains from the Src homology 2/Src homology 3 adaptor Nck. J. Biol. Chem. 274: 27956-27962.

Sparks, A. B., Hoffman, N. G., McConnell, S. J ., Fowlkes, D. M., and Kay, B. K. (1996). Cloning of ligand targets: Systematic isolation of SH3 domain-containing proteins. Nat. Biotechnol. 14: 741-744.

Wang, B., Golemis, E. A., and Kruh, G. D. (1997). ArgBP2, a multiple Src homology 3 domain-containing, Arg/Abl-interacting protein, is phosphorylated in v-Abl-transformed cells and localized in stress fibers and cardiocyte Z-disks. J . Biol. Chem. 272: 17542-17550.

Wang, J. Y. (1993). Abl tyrosine kinase in signal transduction and cell-cycle regulation. Curr. Opin. Genet. Dev. 3: 35- 43. 